\title{
Systematic Handoffs to Improve Patient Outcomes and Resident Satisfaction: a Single-Center Observational Study
}

\author{
Navneet Singh, MD, Christine Yun, MD, Michelle Likhtshteyn, MD, Nirmal Pathak, MD, \\ Steven Massad, MD, Rovie Mesola, MD, and Miguel Ramirez, MD
}

Department of Internal Medicine, SUNY Downstate Medical Center, Brooklyn, NY, USA.

J Gen Intern Med 34(7):1092-3

DOI: $10.1007 / \mathrm{s} 11606-019-04842-\mathrm{w}$

(c) Society of General Internal Medicine 2019

\section{INTRODUCTION}

Medical residents are at the forefront of patient care in many healthcare institutions and thus an integral part of ensuring safe and efficient handoffs. Recent studies have shown that an increase in patient handoffs directly contributes to increased adverse medical events. ${ }^{1,5}$ Implementation of an innovative standardized handoff procedure minimizes medical errors without significantly disrupting workflow. ${ }^{6}$ The purpose of this study was to prospectively assess the belief that a standardized handoff procedure at our institution would improve resident satisfaction while decreasing adverse events.

\section{METHODS}

Residents were asked to track non-lethal adverse events for 6 weeks, which could have been prevented with a more efficient handoff, e.g., an inappropriate altered mental status workup on a patient with known dementia that was not documented and not conferred in the handoff. We then created a standardized handoff procedure with specific details that needed to be conveyed during the handoff. These details included an updated summary of the patient's medical problems and reason for admission, recent patient events (e.g., procedures, change in hemodynamic stability), baseline mental status or cognitive functioning, illness severity (stable, "watcher," or sick), an action list for what needed to be done overnight, and lastly a contingency plan for any perceived events that could occur overnight (e.g., broadening antimicrobial coverage for potentially septic patients). Adverse events were tracked and categorized again after the intervention for another 6 weeks. Previous handoff studies were used to calculate an appropriate sample size. Resident satisfaction with the quality of handoffs for 6 weeks before and after the intervention among Internal Medicine residents on the inpatient medicine service at our university hospital was collected using a three-question survey of satisfaction as rated on a 5-point Likert scale.

Published online February 7, 2019
RESULTS

After initiation of a standardized handoff method, residents were more satisfied with the quality of patient handoffs while covering teams felt more confident managing patients (Table 1). There was a significant reduction in adverse events in three domains of communication (patient summary, illness severity, and action list), but not in the other domains (satisfaction with the patient's summary, list of events, and contingency planning; Table 2).

\section{DISCUSSION}

After implementing a standardized handoff procedure, we saw an increase in resident satisfaction with written and verbal handoffs as well as confidence in residents' ability to care for patients during transitions of care in the inpatient setting. We also found a decrease in non-lethal adverse events in three domains (patient summary, illness severity and action list), with a trend towards improvement in all six domains.

In an era where metrics such as length of stay, infection control parameters, and readmission rates play important roles, it is important to develop systems that streamline the patient's hospital course. There is increasing data that standardizing handoffs and improving transitions in care are imperative to ensure patient safety. Optimizing the handoff process has become a national priority and training programs are obligated to teach learners the skills necessary to ensure safe and efficient transitions in care. ${ }^{2-4}$

Our study limitations include being a single study site and being inadequately powered for three of our outcomes, outcomes that had potentially clinically meaningful decreases. In addition, we did not collect information on unnecessary time spent finding patient information or resources expended performing unnecessary tests. It is likely, based on the decrease in adverse events, that we could have had meaningful improvements in clinical efficiency.

Many institutions are opting for advanced electronic software solutions to help streamline their handoff process, however in our study, we show that by training clinicians on a simple conceptual framework, we can improve satisfaction as well as patient outcomes. Given the increasing importance of improving patient handoffs in all aspects of their medical care, we suggest applying a similar framework to minimize gaps in 
Table 1 Mean satisfaction data. All satisfaction scores ranked on 5-point Likert scales

\begin{tabular}{llr}
\hline \hline & Pre-intervention mean & Post-intervention mean \\
\hline Satisfaction with written handoff & 3.88 & 4.53 \\
Satisfaction with verbal handoff & 3.99 & 4.45 \\
Satisfaction/confidence with patient care & 4.08 & 4.52 \\
\hline
\end{tabular}

Table 2 Mean daily rate of non-lethal adverse events by category

\begin{tabular}{llll}
\hline \hline & Pre-intervention mean & Post-intervention mean & $\boldsymbol{p}$ value \\
\hline Summary & 0.54 & 0.28 & 0.10 \\
Event & 0.28 & 0.15 & 0.35 \\
Baseline & 0.32 & 0.04 & 0.01 \\
Severity & 0.44 & 0.04 & 0.002 \\
Action list & 0.59 & 0.18 & 0.006 \\
Contingencies & 0.68 & 0.32 & 0.09 \\
\hline
\end{tabular}

patient care and reduce the burden on the healthcare system. Standardizing handoffs has the potential to positively impact healthcare.

Acknowledgments: The investigators would like to acknowledge Jay Thompson for his tireless work in revising the handoff software to accommodate our study. We would also like to thank the internal medicine residents who devoted their limited time to participation in this study.

Corresponding Author: Navneet Singh, MD; Department of Internal Medicine SUNY Downstate Medical Center, Brooklyn, NY, USA (e-mail: navneet.singh@downstate.edu).

\section{Compliance with Ethical Standards:}

Conflict of Interest: The authors declare that they do not have a conflict of interest.
Publisher's Note: Springer Nature remains neutral with regard to jurisdictional claims in published maps and institutional affiliations.

\section{REFERENCES}

1. Abraham J, Kannampallil T, Patel VL. A systematic review of the literature on the evaluation of handoff tools: implications for research and practice. J Am Med Inform Assoc. 2013;21(1):154-162.

2. The Joint Commission. Inadequate hand-off communication. Sentinel Event Alert. 2017; 58:1-6

3. Revere, A., Eldridge, N. Joint Commission National Patient Safety Goals for 2008. Topics in Patient Safety. 2008:12(1):1-4.

4. The Joint Commission Center for Transforming Healthcare. Improving Transitions of Care: Hand-Off Communications. 2014.

5. Kitch BT, Cooper JB, Zapol WM, et al. Handoffs Causing Patient Harm: A Survey of Medical and Surgical House Staff. Jt Comm J Qual Patient Saf. 2008;34(10):563-570d.

6. Starmer AJ, Spector ND, Srivastava R, et al. Changes in medical errors after implementation of a handoff program. N Engl J Med. 2014;371(19):1803-1812. 